\title{
The planet-hosting subdwarf B star V 391 Pegasi is a hybrid pulsator ${ }^{\star}$
}

\author{
R. Lutz ${ }^{1,4}$, S. Schuh ${ }^{1}$, R. Silvotti ${ }^{2}$, S. Bernabei ${ }^{3}$, S. Dreizler ${ }^{1}$, T. Stahn ${ }^{4}$, and S. D. Hügelmeyer ${ }^{1}$ \\ ${ }^{1}$ Institut für Astrophysik, Universität Göttingen, Friedrich-Hund-Platz 1, 37077 Göttingen, Germany \\ e-mail: rlutz@astro.physik.uni-goettingen.de \\ 2 INAF - Osservatorio Astronomico di Capodimonte, via Moiariello 16, 80131 Napoli, Italy \\ INAF - Osservatorio Astronomico di Bologna, via Ranzani 1, 40127 Bologna, Italy \\ 4 Max-Planck-Institut für Sonnensystemforschung, Max-Planck-Straße 2, 37191 Katlenburg-Lindau, Germany
}

Received 17 December 2007 / Accepted 19 December 2008

\section{ABSTRACT}

\begin{abstract}
Context. A noticeable fraction of subdwarf B stars shows either short-period ( $p$-mode) or long-period ( $g$-mode) luminosity variations, with two objects so far known to exhibit hybrid behaviour, i.e. showing both types of modes at the same time. The pulsating subdwarf B star V 391 Pegasi (or HS 2201+2610), which is close to the two known hybrid pulsators in the $\log g-T_{\text {eff }}$ plane, has recently been discovered to host a planetary companion.

Aims. In order to learn more about the planetary companion and its possible influence on the evolution of its host star (subdwarf B star formation is still not well understood), an accurate characterisation of the host star is required. As part of an ongoing effort to significantly improve the asteroseismic characterisation of the host star, we investigate the low-frequency behaviour of HS $2201+2610$. Methods. We obtained rapid high signal-to-noise photometric CCD ( $B$-filter) and PMT (clear-filter) data at $2 \mathrm{~m}$-class telescopes and carried out a careful frequency analysis of the light curves.

Results. In addition to the previously known short-period luminosity variations in the range $342 \mathrm{~s}-367 \mathrm{~s}$, we find a long-period variation with a period of $54 \mathrm{mn}$ and an amplitude of 0.15 per cent. This can most plausibly be identified with a $g$-mode pulsation, so that HS 2201+2610 is a new addition to the short list of hybrid sdB pulsators.

Conclusions. Along with the previously known pulsating subdwarf B stars HS 0702+6043 and Balloon 090100001 showing hybrid behaviour, the new hybrid HS 2201+2610 is the third member of this class. This important property of HS $2201+2610$ can lead to a better characterisation of this planet-hosting star, helping the characterisation of its planetary companion as well. Current pulsation models cannot yet reproduce hybrid sdBV stars particularly well and improved pulsation models for this object have to include the hybrid behaviour.
\end{abstract}

Key words. subdwarfs - stars: horizontal-branch - stars: oscillations - stars: individual: HS 2201+2610

\section{Introduction}

Subdwarf B stars (sdBs) are evolved objects with masses around half a solar mass and are thought to be core helium burning. Having lost most of their original hydrogen envelope in earlier stages of their evolution, the remaining H-rich envelope is too thin to sustain hydrogen shell burning. The sdBs populate the extreme horizontal branch (EHB) at effective temperatures of 20000 to $40000 \mathrm{~K}$ and surface gravities $\log \left(\mathrm{g} / \mathrm{cm} \mathrm{s}^{-2}\right)$ between 5.0 and 6.2. Instead of evolving towards the asymptotic giant branch, the sdBs are predicted to follow tracks leading directly towards the white dwarf region after leaving the extreme horizontal branch. A fraction of the sdB stars shows pulsations: the first pulsating sdB star was discovered in 1997. Variable subdwarf B stars (sdBV stars) can be divided into the classes of rapid p-mode pulsators $\left(\mathrm{sdBV}_{\mathrm{r}}\right)$ and slow g-mode pulsators $\left(\mathrm{sdBV}_{\mathrm{s}}\right)$, with two objects known so far to belong to both classes simultaneously (hybrid pulsators, $\mathrm{sdBV}_{\mathrm{rs}}$ ).

The $p$-mode pulsators show low amplitudes (few ten mmag) and short periods (few minutes) at higher temperatures. The

\footnotetext{
* Based on observations collected at the Centro Astronómico Hispano Alemán (CAHA) at Calar Alto, operated jointly by the Max-Planck Institut für Astronomie and the Instituto de Astrofísica de Andalucía (CSIC).
}

acoustic pressure waves are of low degree and low radial order. In contrast, the $g$-mode pulsators have even lower amplitudes (few mmag) and longer periods (30 to $90 \mathrm{mn}$ ) at lower temperatures. The class prototypes are EC 14026-2647 (Kilkenny et al. 1997) and PG 1716+426 (Green et al. 2003), respectively. Both mode types are thought to be driven by the same $\kappa$-mechanism due to an opacity bump of iron and nickel (Charpinet et al. 1997; Fontaine et al. 2003; Jeffery \& Saio 2006). Hybrid sdB pulsators show $p$ - and $g$-modes simultaneously and are of particular interest since the two mode types probe different regions within the star. In the $\log g-T_{\text {eff }}$ diagram, the hybrids are located at the interface of the $p$-mode and $g$-mode instability regions. The known hybrids are HS 0702+6043 (Schuh et al. 2005, 2006; Lutz et al. 2008) and Balloon 090100001 (Oreiro et al. 2005; Baran et al. 2005).

This paper presents the third member of this group: HS $2201+2610$ is a $14.3 \mathrm{~B}$-magnitude star having an effective temperature of $29300 \pm 500 \mathrm{~K}$ and a surface gravity $\log g$ of $5.40 \pm 0.10$ in cgs units (Østensen et al. 2001). Based on these spectroscopic parameters, which are very similar to those of HS 0702+6043 and Balloon 090100001, HS 2201+2610 was a natural further target to search for simultaneous $g$-modes in a known $p$-mode pulsator after the discovery of the first hybrids. The high signal-to-noise observations initiated to check 


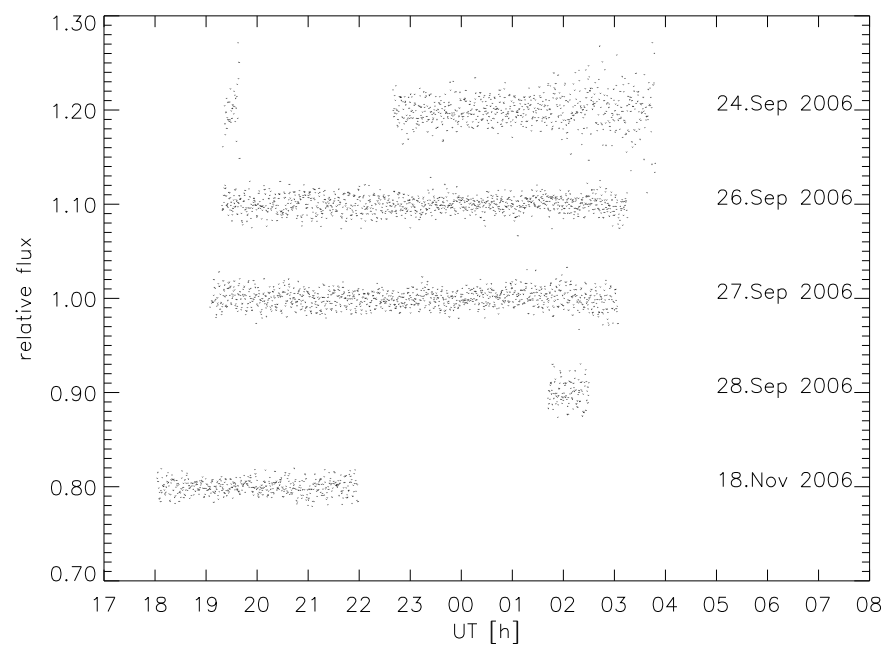

Fig. 1. Light curves from the September/November 2006 runs at Calar Alto. The relative flux is set to 1.0 for the 27. Sep. 2006 light curve and previous (following) nights are shifted upwards (downwards) by 0.1 units.

for hybrid behaviour in HS 2201+2610 fitted in well with our ongoing long-term monitoring in order to search for secular changes in the pulsation periods indicative of slow evolutionary changes in the star (Silvotti et al. 2002), which has recently resulted in the surprising detection of a planetary companion around HS 2201+2610 (better known as V 391 Pegasi b, Silvotti et al. 2007). In fact, from a few of the better-quality data sets obtained within this overall observational effort up to the end of 2005 , we already suspected the presence of additional power in the low-frequency domain of HS 2201+2610.

In Sect. 2 we present the photometric data obtained specifically to investigate the gravity mode frequency range, as well as suitable confirmation data obtained as part of our longterm monitoring. The data reduction and frequency analysis are described in Sect. 3, and as a result of our investigation we will present HS $2201+2610$ as a new member of the group of hybrid pulsating sdB stars. We discuss the implications of this discovery for the ongoing asteroseismic characterisation of HS 2201+2610, in the context of our efforts to constrain the mass of its planetary companion, in Sect. 4. Finally, we present a concluding outlook in Sect. 5.

\section{Observations}

The light curves (see Fig. 1) were obtained at Calar Alto Observatory in September and November 2006. The instrumental setup was chosen to consist of the Calar Alto Faint Object Spectrograph (CAFOS) focal reducer attached to the $2.2 \mathrm{~m}$ telescope. A $2 \mathrm{k} \times 2 \mathrm{k}$ CCD chip recorded the science frames through a Johnson $B$ filter with a $2 \times 2$ binning and an exposure time of ten seconds with an average cycle time of $25.5 \mathrm{~s}$. In order to sample the short-period $p$-modes as well as to detect very lowamplitude $g$-modes, one has to find a good compromise with the exposure time. Ten seconds turned out to be sufficient to resolve the $p$-mode cycles as well as provide a $\mathrm{S} / \mathrm{N}$ good enough to resolve low-amplitude signals. We have a total of about $26 \mathrm{~h}$ of photometric data.

The data reduction was carried out using the time resolved imaging photometry package (TRIPP, see Schuh et al. 2003), which is designed to handle the aperture photometry of large CCD sets. This includes bias-, flatfield- and background corrections and finally yields extracted light curves in the form of relative flux (in comparison to four stable reference stars) as a function of geocentric Julian date. Additionally, a barycentric time correction was applied. Differential extinction effects on a night-by-night basis were corrected using second-orderpolynomials.

In addition to our Calar Alto data from 2006, one of us (R. S.) provided older data from August/September 2003, which were part of the whole earth telescope extended coverage 23 (WET Xcov23). The data taken at Loiano Observatory (LOI, $1.5 \mathrm{~m}$ telescope and 3 channel photometer) and Observatoire de HauteProvence (OHP, $1.9 \mathrm{~m}$ telescope and 3 channel photometer) were provided as extracted light curves (total length of $28 \mathrm{~h}$ ) and we used them to cross-check our Calar Alto results.

\section{Results}

We carried out a frequency analysis on both data sets with a program called sinfit, which is part of TRIPP. This includes leastsquares non-linear sinusoidal fits to the light curves and calculation of the corresponding periods, amplitudes and phases. The amplitudes are given in milli modulation intensity units (mmi). In this context $10 \mathrm{mmi}$ means that the luminosity has changed by one per cent with respect to the mean brightness level. We used the Scargle algorithm (Scargle 1982) to calculate lombscargle-periodograms (LSPs) in the form of power spectral density (psd) as a function of frequency, together with confidence levels, which are based on false alarm probabilities (faps). See Sect. 4 in Dreizler et al. (2002) for a more detailed description. In our case, we derived the faps from 158000 white noise simulations. This yields a confidence level of $4 \sigma$, judging a signal above this level to be real with a probability of $99.99366 \%$.

Table 1 summarizes the results of our frequency analyses of both data sets. We refer to the pulsation frequency identifications obtained by Silvotti et al. (2002) and also list the mode degree $(l)$ identification suggested there in Fig. 9. For the Calar Alto data we estimate the errors in the frequency to be $0.2 \mu \mathrm{Hz}$ (FWHM of the central peak in the window function) and having a detection limit of $1.1 \mathrm{mmi}$, which was calculated as four times the mean noise level in the residual DFT. We used (f4) and (f5) in the prewhitening, because low-amplitude signals at these frequency ranges were also found in other runs (Silvotti et al. 2002). Regarding the OHP/LOI data, we calculate a frequency resolution of $0.54 \mu \mathrm{Hz}$ (FWHM of the central peak in the window function) and a detection limit of $0.97 \mathrm{mmi}$ (four times the mean noise level in the residual DFT). From the WET Xcov23 runs, the OHP data from August 22/23 and the LOI data from August 29/31 and September 3/4 have been included. The frequencies of the "mean" solution refer to BJD 2452788, which corresponds to the middle of the 7-year long data set in Silvotti et al. (2007). Estimating the formal frequency resolution of the 7-year "mean" solution yields $0.0045 \mu \mathrm{Hz}$.

We can clearly recover the three strongest modes found in an extensive run published by Silvotti et al. (2002), here labeled with $\mathrm{f} 1$ to $\mathrm{f} 3$. These frequencies are assigned to pressure driven modes. The original and residual LSPs of our Calar Alto data in the $p$-mode regime are displayed in Fig. 2. In addition, we find a long-period luminosity variation (f7, shown in Fig. 3) of about $54 \mathrm{mn}$.

As mentioned before, we looked at suitable WET Xcov23 data with the focus on the $g$-mode domain to confirm our detection of a long-period luminosity variation found in the Calar Alto data (left plots in Fig. 3). The periodogram of these additional data is shown in the right plots of Fig. 3. The OHP/LOI 
Table 1. Results of our frequency analysis of HS 2201+2610.

\begin{tabular}{|c|c|c|c|c|c|c|c|c|c|}
\hline \multirow[b]{2}{*}{ ID } & \multirow[b]{2}{*}{ Type } & \multirow[b]{2}{*}{$l$} & \multirow[b]{2}{*}{$\begin{array}{l}\text { Period } \\
\text { [s] }\end{array}$} & \multicolumn{2}{|c|}{ Calar alto data (2006) } & \multicolumn{2}{|c|}{ OHP/LOI Data (2003) } & \multicolumn{2}{|c|}{ Mean solution (2000-2007) } \\
\hline & & & & $\begin{array}{l}\text { Frequency } \\
{[\mu \mathrm{Hz}]}\end{array}$ & $\begin{array}{l}\text { Amplitude } \\
\text { [mmi] }\end{array}$ & $\begin{array}{l}\text { Frequency } \\
{[\mu \mathrm{Hz}]}\end{array}$ & $\begin{array}{l}\text { Amplitude } \\
\text { [mmi] }\end{array}$ & $\begin{array}{l}\text { Frequency } \\
{[\mu \mathrm{Hz}]}\end{array}$ & $\begin{array}{l}\text { Amplitude } \\
\text { [mmi] }\end{array}$ \\
\hline f1 & $p$ & $\overline{0}$ & 349.5 & 2861.2 & 6.6 & 2860.9 & 12.3 & 2860.9387 & 10.4 \\
\hline $\mathrm{f} 2$ & $p$ & 1 & 354.2 & 2823.7 & 3.3 & 2824.1 & 3.7 & 2824.0965 & 4.2 \\
\hline f3 & $p$ & 1 & 347.1 & 2880.8 & 1.5 & 2880.7 & 1.5 & 2880.6842 & 0.7 \\
\hline $\mathrm{f} 4$ & $p$ & 2 & & & & & & 2921.8463 & 0.6 \\
\hline$(\approx \mathrm{f} 4$ alias $)$ & & & 344.1 & 2906.3 & 0.4 & & & & \\
\hline $\begin{array}{c}\text { (f5) } \\
\text { (f5 alias) }\end{array}$ & $p$ & 2 & 366.9 & 2725.9 & 0.8 & & & & \\
\hline (f6) & $p$ & & & & & & & $(2882.0039$ & $0.5)$ \\
\hline f7 & $g$ & & 3255.6 & 307.2 & 1.5 & 306.8 & 1.5 & & \\
\hline
\end{tabular}
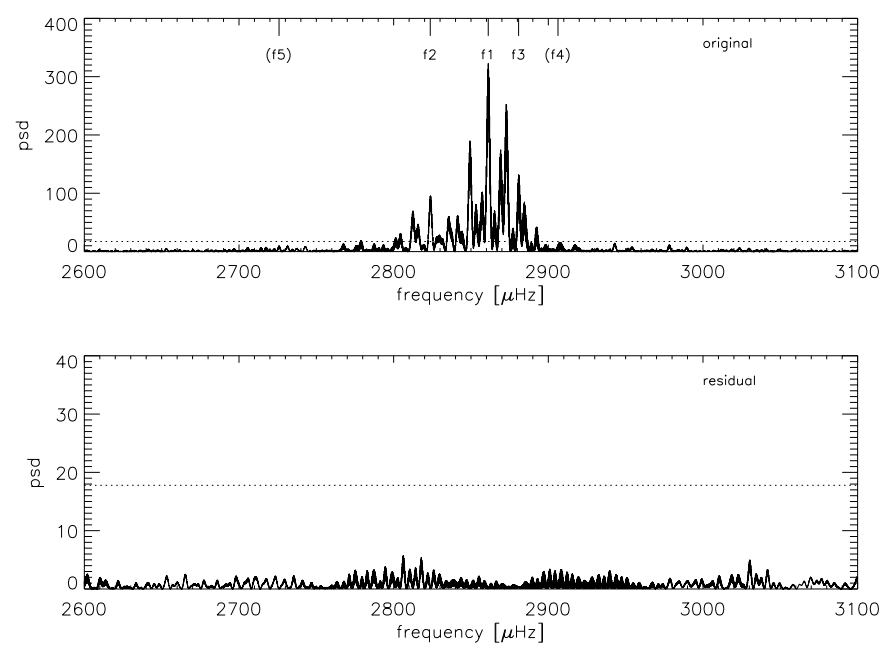

Fig. 2. Lomb Scargle Periodograms (LSPs) of the $p$-mode regime. The top panel shows the complete Calar Alto data. The bottom panel displays the LSP after simultaneous subtraction of five frequencies. Horizontal dotted lines indicate $4 \sigma$ confidence levels and the marks correspond to the IDs in Table 1.

results (also in Table 1) clearly confirm the detection of a low frequency oscillation in HS 2201+2610.

As can be seen in the middle right plot in Fig. 3, there is still further residual power above $4 \sigma$ in the OHP/LOI data LSP after subtraction of the long-period variation $\mathrm{f7}$. However, this is not seen in the Calar Alto data (which were taken three years later) so that further high-quality data are required to test the persistence of these residual peaks.

\section{Discussion}

We identify the long-period low-amplitude luminosity variation in HS $2201+2610$ with a gravity driven mode. The presence of both types of modes within HS 2201+2610 would therefore define this object to be a member of the hybrid $\mathrm{sdB}$ pulsator group, beside the previously known hybrids HS $0702+6043$ and Balloon 090100001. Other interpretations for the long-period variation can be excluded with a high probability with similar arguments as for HS 0702+6043 (Schuh et al. 2006). The same long-period variation, in frequency as well as amplitude, is present in data sets three years apart. This rules out sky or transparency variations, as well as rotating star spots. The fast rotation rate that would be required for the latter interpretation is further unlikely since rotation periods of single sdB stars are typically much longer. Rotational splitting (for $l>0$ modes) has not yet been detected in HS 2201+2610, but the split components often have lower amplitudes and might be below our detection limit. With the light-travel time effect used in the detection of the planet candidate around HS 2201+2610 in its wide orbit, we would not be sensitive to a further hypothetical companion in a very close orbit with a $54 \mathrm{mn}$ period. A binary origin of the long-period variation is unlikely for the following reasons. A $54 \mathrm{mn}$ orbital period would be at the extreme low limit of the observed and theoretical orbital period distributions (see e.g. Fig. 2 in Morales-Rueda et al. 2006). Additionally, a low-mass main-sequence (M-dwarf) companion as the source of the $54 \mathrm{mn}$ signal can be excluded by geometrical considerations (Roche-Limits vs. separation in Keplerian orbits). The same is true for a $\mathrm{sdB}+\mathrm{WD}$ configuration in a $54 \mathrm{mn}$ orbit down to very low WD-masses (below $0.2 M_{\odot}$ ). In the case of deformation of the $\mathrm{sdB}$ by a hypothetical WD companion, causing ellipsoidal variation, the underlying orbital period would be $108 \mathrm{mn}$, but we would then expect a signal at least ten times stronger down to low inclinations, dropping to the observed very low amplitude at only about $10^{\circ}$. A WD companion as the source of the signal is therefore also very unlikely. For less massive and hence harder to detect companions it would be increasingly hard to prevent mass transfer in the system. Binary orbital motion as the cause of the long-period variation will immediately and conclusively be ruled out if its multi-periodicity is confirmed.

The period and amplitude fall within the typical range for $g$ mode sdB pulsations, strongly suggesting that the variation is in fact due to a $g$-mode. Finally, in comparison to the location of the other two known hybrid sdB pulsators in the $\log g-T_{\text {eff }}$ diagram of sdBV stars (see e.g. Fig. 1 in Lutz et al. 2008), the very similar parameters of HS 2201+2610 again imply that hybrid behaviour may plausibly be expected. All three objects are located at the interface between the empirical $p$-mode and $g$-mode instability regions. Hybrid pulsators have also been shown to exist in the partially overlapping regions of the $\beta$ Cep / [SPB] stars, which resemble the $p / g$-mode sdB pulsators in many ways (e.g. Handler et al. 2004; Jerzykiewicz et al. 2005; Handler et al. 2006; Pigulski \& Pojmański 2008a,b). Hybrid behaviour is also observed in the less closely related $\delta$ Sct / $\gamma$ Dor stars (e.g. Henry \& Fekel 2005).

From a theoretical point of view, modeling $p$-mode sdB pulsators has been very successful. Fontaine et al. (2006) report on the determination of the basic structural parameters of twelve $p$-mode sdB pulsators. Regarding the $g$-mode sdB pulsators there are still issues. The "second-generation models" presented by Charpinet et al. (1997) and Fontaine et al. (2003) diverge by some $4000 \mathrm{~K}$ from the observations, i.e. they fall short of the empirical blue edge of the $g$-mode pulsators. A solution to this problem has been suggested by Jeffery \& Saio (2006, 2007): In their models, using opacity project (OP) instead of OPAL 

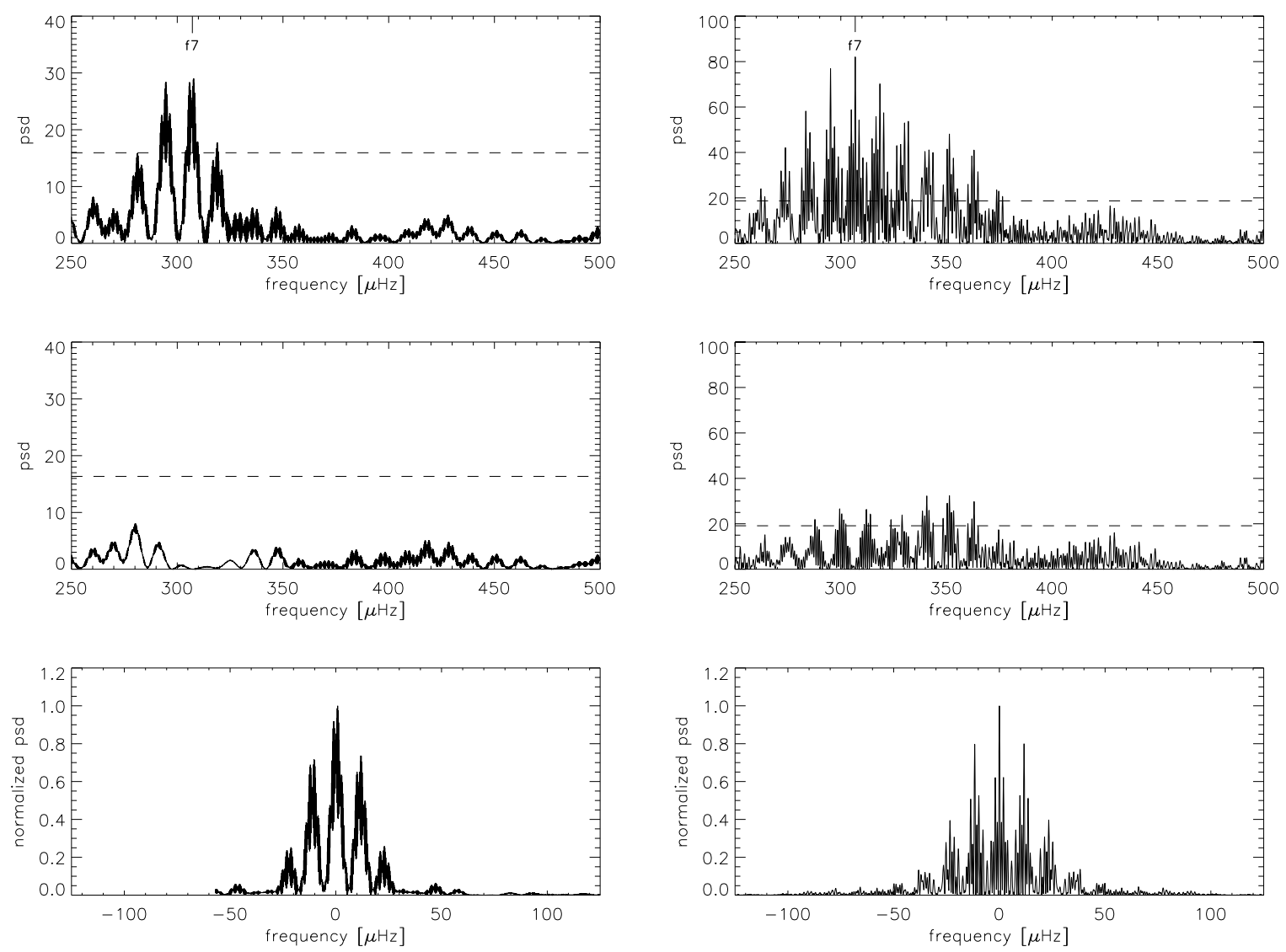

Fig. 3. Results of our frequency analysis in the $g$-mode regime. Left: September/November 2006 Calar Alto data. The top panel is a Lomb Scargle Periodogram of the three best nights of the full light curve where five frequencies have already been subtracted (f1 to f5, see Table 1). Fitting a long-periodic luminosity variation (f7) to this subset of the data results in the residual LSP displayed in the middle. The window function is shown in the bottom panel. Right: August/September 2003 OHP/LOI data. The top panel displays the Lomb Scargle Periodogram of the light curve after pre-whitening of three frequencies ( $\mathrm{f} 1$ to $\mathrm{f} 3$, see Table 1). The residual LSP after subtraction of the long-period variation $\mathrm{f} 7$ is shown in the middle and the window function is illustrated in the bottom panel. Horizontal dashed lines indicate $4 \sigma$ confidence levels.

opacity tables and including $\mathrm{Ni}$ in addition to $\mathrm{Fe}$ to the opacity profile shifts the theoretical blue edge close to the empirical one. Both aspects of this opacity problem are, in close analogy, also relevant for the $\beta \mathrm{Cep}$ and [SPB] pulsators (Miglio et al. 2007). In fact, the hybrid objects (both in the main-sequence as well as in the extreme horizontal branch pulsators groups) in particular represent excellent test cases for the adequateness of the opacities used, and should provide strong constraints. From previous experience, it appears to be clear that radiative levitation and self-consistent diffusion of the $\mathrm{Fe} / \mathrm{Ni}$ group elements need to be included in the EHB models. It will be interesting to see if "third-generation models" incorporating both radiative levitation/diffusion and improved opacities will finally deliver an adequate description of the hybrid sdB pulsators while still reproducing the behaviour of the $p$-mode pulsators as consistently as before, and at the same time correctly describing the extent of the empirical $g$-mode instability region.

\section{Concluding outlook}

As recently published by Silvotti et al. (2007), HS 2201+2610 has a planetary companion which was detected using asteroseismological techniques. Long-term monitoring of the strongest $p$-mode pulsations made it possible to derive secular variations of the two main pulsation frequencies, leading to an evolutionary timescale of around 5-8 $\times 10^{6}$ years, which matches theoretical evolutionary models of extreme horizontal branch stars. Periodic residuals in the $O-C$ diagram (observed - calculated) analysis, attributable to light-travel time effects, revealed the presence of an $m \sin i=3.2 M_{\text {Jup }}$ companion around HS 2201+2610 in a $3.2 \mathrm{yr}$ orbit. We are in the process of continuing our regular long term photometric monitoring of HS $2201+2610$ beyond the current coverage of two orbit cycles. In order to further characterise the companion, especially to determine its mass, it is necessary to constrain the orbital inclination of the system and to derive a more accurate asteroseimic mass of the host star.

The mass of the host star is in principle accessible through asteroseismology, and Silvotti et al. (2002) already presented one possible solution. The determination of the fundamental parameters of the star has however only weakly been constrained in that work due to the small number of modes detected. Now that we have added HS $2201+2610$ to the list of hybrid sdB pulsators, this finding might provide the additional constraints necessary for a reliable and accurate asteroseismic characterisation. At this point, we emphasize again the current need to improve on the model calculations first. For the individual object HS 2201+2610, it will be a special challenge to additionally drive $g$-modes in an improved version of one of the possible models found by Silvotti et al. (2002). 
Acknowledgements. The Loiano and OHP data were taken during the Whole Earth Telescope extended coverage run 23; we thank the WET Xcov23 team: R. Janulis, J.-E. Solheim, R. Østensen, T. D. Oswalt, I. Bruni, R. Gualandi, A. Bonanno, G. Vauclair, M. Reed, C.-W. Chen, E. Leibowitz, M. Paparo, A. Baran, S. Charpinet, N. Dolez, S. Kawaler, D. Kurtz, P. Moskalik, R. Riddle, S. Zola. Part of the observations at Calar Alto were carried out in service mode by U. Thiele and A. Guijarro. The observations at Calar Alto were supported through Deutsche Forschungsgemeinschaft (DFG) travel grant SCHU 2249/3-1.

\section{References}

Baran, A., Pigulski, A., Kozieł, D., et al. 2005, MNRAS, 360, 737 Charpinet, S., Fontaine, G., Brassard, P., et al. 1997, ApJ, 483, L123 Dreizler, S., Schuh, S., Deetjen, J. L., Edelmann, H., \& Heber, U. 2002, A\&A, 386,249

Fontaine, G., Brassard, P., Charpinet, S., et al. 2003, ApJ, 597, 518

Fontaine, G., Brassard, P., Charpinet, S., et al. 2006, in Proceedings of SOHO 18/GONG 2006/HELAS I, Beyond the spherical Sun, ESA SP, 624

Green, E. M., Fontaine, G., Reed, M. D., et al. 2003, ApJ, 583, L31

Handler, G., Jerzykiewicz, M., Rodríguez, E., et al. 2006, MNRAS, 365, 327 Handler, G., Shobbrook, R. R., Jerzykiewicz, M., et al. 2004, MNRAS, 347, 454 Henry, G. W., \& Fekel, F. C. 2005, AJ, 129, 2026
Jeffery, C. S., \& Saio, H. 2006, MNRAS, 372, L48

Jeffery, C. S., \& Saio, H. 2007, MNRAS, 378, 379

Jerzykiewicz, M., Handler, G., Shobbrook, R. R., et al. 2005, MNRAS, 360, 619

Kilkenny, D., Koen, C., O’Donoghue, D., \& Stobie, R. S. 1997, MNRAS, 285, 640

Lutz, R., Schuh, S., Silvotti, R., et al. 2008, in Hot Subdwarf Stars and Related Objects, ed. U. Heber, C. S. Jeffery, \& R. Napiwotzki, ASP Conf. Ser., 392, 339

Miglio, A., Montalbán, J., \& Dupret, M.-A. 2007, MNRAS, 375, L21

Morales-Rueda, L., Maxted, P. F. L., Marsh, T. R., Kilkenny, D., \& O’Donoghue, D. 2006, Baltic Astron., 15, 187

Oreiro, R., Pérez Hernández, F., Ulla, A., et al. 2005, A\&A, 438, 257

Østensen, R., Solheim, J.-E., Heber, U., et al. 2001, A\&A, 368, 175

Pigulski, A., \& Pojmański, G. 2008a, A\&A, 477, 907

Pigulski, A., \& Pojmański, G. 2008b, A\&A, 477, 917

Scargle, J. D. 1982, ApJ, 263, 835

Schuh, S., Dreizler, S., Deetjen, J. L., \& Göhler, E. 2003, Baltic Astron., 12, 167 Schuh, S., Huber, J., Dreizler, S., et al. 2006, A\&A, 445, L31

Schuh, S., Huber, J., Green, E. M., et al. 2005, in 14th European Workshop on White Dwarfs, ed. D. Koester, \& S. Moehler, ASP Conf. Ser., 334, 530

Silvotti, R., Janulis, R., Schuh, S., et al. 2002, A\&A, 389, 180

Silvotti, R., Schuh, S., Janulis, R., et al. 2007, Nature, 449, 189 